\title{
Adaptive Coordinated Motion Constraint Control for Cooperative Multi-manipulator Systems
}

\section{Chunjian Su}

Shandong University of Science and Technology

\section{Min Zhang}

Shandong University of Science and Technology

Shuai Zhang

Shandong University of Science and Technology

Sumin Guo ( $\nabla$ sdkjjd314@163.com )

Shandong University of Science and Technology

\section{Rui Wang}

Shandong University of Science and Technology

\section{Guosong Zhang}

Shandong University of Science and Technology

\section{Yanan Yao}

Shandong University of Science and Technology

\section{Qiang Zhang}

Shandong University of Science and Technology

\section{Research Article}

Keywords: Multi-manipulator cooperation, Collaboration system, Adaptive coordination, Kinematic constraints

Posted Date: September 7th, 2021

DOl: https://doi.org/10.21203/rs.3.rs-780724/v1

License: (c) (i) This work is licensed under a Creative Commons Attribution 4.0 International License. Read Full License

Version of Record: A version of this preprint was published at The International Journal of Advanced Manufacturing Technology on January 11th, 2022. See the published version at https://doi.org/10.1007/s00170-021-08621-y. 


\title{
Title page
}

\section{Title: Adaptive coordinated motion constraint control for cooperative multi-manipulator systems}

\author{
Author:Chunjian Su,Min Zhang,ShuaiZhang,Sumin Guo* (Corresponding Author \\ ) , Rui Wang,Guosong Zhang, Yanan Yao,Qiang Zhang \\ E-mail: sdkjjd314@163.com
}

\section{Author information}

Chunjian Su, man, Shandong Province of China, Chinese,

Professor, School of mechanical and electronic engineering, Shandong University of science and technology.

I have presided over or participated in more than 30 research projects and published more than 50 academic papers, of which 30 were searched by SCI and EI, and applied for more than 60 national patents, ofwhich more than 20 were invention patents. 


\begin{abstract}
A cooperative multi-manipulator system is a nonlinear, time-varying, strong coupling system with multiple inputs and outputs. Because of external disturbance, load change and other factors, a cooperative multi-manipulator system needs to address the problems of uncertain environment interaction, feasible control methods and coordinated planning. In this study, the coordinated motion of a multi-manipulator is divided into two types: coupling and superposition motions. An adaptive coordinated motion constraint scheme is proposed for the two different motion forms. The coupled and superposition motions were investigated by coordinating a carrying task and a circle drawing task, respectively, and the effectiveness of the adaptive coordinated motion scheme was verified. Based on the adaptive kinematics constraint algorithm of the multi-manipulator, co-simulation analysis of the multi-manipulator coordinated motion is conducted. Simulation results show that the multi-manipulator preserves the coordination relationship at all points of the trajectory planning path and has a good motion effect. Finally, an experimental platform for a cooperative multi-manipulator system was built to conduct experimental research on the coordinated motion of the multi-manipulator. Experimental results show that the proposed adaptive motion constraint control scheme for cooperative multi-manipulator systems has a good coordination effect, strong adaptivity and high-control precision.
\end{abstract}

Keywords: Multi-manipulator cooperation; Collaboration system; Adaptive coordination; Kinematic constraints. 


\section{Introduction}

One of the trending topics in manipulator research is the coordinated motion of multiple manipulators ${ }^{[1][2]}$. The emergence of cooperative multi-manipulator systems overcomes the working limit of a single manipulator system by providing larger working space, greater working ability, a more flexible system structure and greater load capacity compared with a single manipulator. Cooperative multi-manipulator systems can accomplish many difficult tasks that cannot be accomplished by a single manipulator through coordinated movement ${ }^{[3][4]}$, such as coordinated handling and welding. Multi-manipulator cooperation has many advantages over a single manipulator; however, it also introduces many control issues. Compared with a single manipulator, a cooperative multi-manipulator system has a more complex organisational structure, which is not a simple combination of multiple manipulators. It needs to address the problems of uncertain environment interaction, feasible control methods and coordinated planning ${ }^{[5]}$. Multi-manipulator coordination involves the cross-integration of multiple disciplines and is extremely complicated to implement. When multiple manipulators operate an object together, a closed chain system will be formed, which inevitably leads to control problems such as redundant degrees of freedom and motion limitation. Therefore, research into the motion coordination of multiple manipulators is required ${ }^{[6-9]}$.

Zhang Haitao and Tang Licai proposed a path planning algorithm for spatial multi-manipulator coordinated motion depending on whether there is a relative motion in the process of multi-manipulator coordinated motion ${ }^{[10]}$. Hou Y and Wang TA proposed a welding path planning method by studying path planning problems of dual-robot coordinated welding of complex spatial welds on the basis of a multi-objective genetic algorithm ${ }^{[11]}$. Zhang Tstudied the welding coordination problem of two robots for complex curve welding. The master/slave scheme was used for coordinated motion planning, and motion trajectories of two manipulators were automatically calculated through a constraint relationship ${ }^{[12]}$. Park Ha and Lee C described the coordinated motion of two manipulators and investigated how to effectively use motion variables to control the coordinated motion of two manipulators ${ }^{[13]}$. Davidson J R proposed a coordinated motion planning method for two manipulators by combining evolutionary and local search algorithms ${ }^{[14]}$. Wang $X$ and Chen $\mathrm{L}$ conducted theoretical derivation and experimental research on the coordinated movement of dual manipulators. From the perspective of visual information processing, they analysed and solved the specific target object recognition process. On this basis, a complete set of coordinated movements was proposed control ${ }^{[15]}$. The ability to perform coordinated movements is the premise of multi-manipulator collaboration. Existing multi-manipulator coordinated motion research is scarce, and the coordination effect is poor, often only solving relatively simple coordination tasks. For complex coordination tasks, existing coordination control schemes have poor coordination effects ${ }^{[16][17]}$. Thus, a multi-manipulator coordinated motion control scheme with high coordination accuracy and good coordination effect is urgently needed. 
Aiming at the problem of the coordinated motion of cooperative multi-manipulator systems, this study divides the coordinated motion of multi-manipulator systems into two motion forms: coupling and superposition motions.An adaptive coordinated motion constraint scheme is proposed for the two different motion forms. The coupling and superposition motions are investigated by coordinating transportation and circle drawing tasks, respectively. A robot toolbox is used to verify two kinematic constraint control schemes. To simulate the process of multi-manipulator coordinated motion more realistically, the MATLAB-ADAMS Co-simulation analysis of multi-manipulator coordinated motion is conducted, and the dynamic simulation of coupling and superposition motions of cooperative multi-manipulator systems is realised. Finally, the correctness of the algorithm is verified by experimenting with two $6 \mathrm{R}$ manipulators for coordinated motion.

\section{Adaptive trajectory coordination motion constraint control of multi-manipulator}

Multi-manipulator cooperation has many advantages, such as large workspace, low load performance requirements and the ability to complete more complex operation tasks, and the coordinated movement of multi-manipulators is the key to realise multi-manipulator cooperation ${ }^{[18]}$. Before the trajectory coordination planning of a cooperative multi-manipulator system, the kinematics constraint relations of the cooperative multi-manipulator system should be classified ${ }^{[19]}$, and different constraint relations have different trajectory coordination methods. According to different kinematics constraint relations of the cooperative multi-manipulator system, the coordinated motion of the system can be divided into two categories:

(1) Coupled motion of multi-manipulators: there is a specific pose constraint relationship between the reference points of the end-effector of multi-manipulators, and through this specific kinematic constraint relationship, the pose between the end-effector of the manipulators remains unchanged.

(2) Multi-manipulator superposition motion: there is not only a specific pose constraint relationship between the end-effector reference points of multi-manipulators but also a relative motion constraint relationship, and the final motion trajectory is the superposition of the two parts.

\subsection{Coordinated constraint control of multi-manipulator coupling motion}

Multi-manipulator handling is the most typical type of multi-manipulator coupling motion. When multi-manipulator coupling motion planning is conducted for two manipulators, one of the manipulators is determined as the master manipulator, and the other as the slave manipulator. The relative pose between the slave manipulator and the end-effector of the master manipulator should be kept unchanged during movement. Since the slave manipulator follows the master manipulator, it is not necessary to perform separate trajectory planning for the slave manipulator. After 
the trajectory of the end-effector reference point of the master manipulator is determined, the unique trajectory of the slave manipulator can be obtained according to the coupling kinematics relationship.

\subsubsection{Coupled motion constraint algorithm}

Two reference points $T_{1}$ and $T_{2}$ at different positions were selected on the trajectory of the main manipulator, ${ }^{A} T_{1}$ and ${ }^{A} T_{2}$ represent the coordinates of the trajectory point and the trajectory point in the base coordinate system of the multi-manipulator. Assuming that the main manipulator moves from track points $T_{1}$ to $T_{2}$, the slave manipulator must move from track points $T_{1}$ to $T_{2}$. According to the definition of coupling motion, the relative pose relation between track points $T_{1}$ and $T_{\mathrm{I}}$ is equal to that between track points $T_{2}$ and $T_{\mathrm{II}}$. Let ${ }^{B} T_{\mathrm{I}}$ and ${ }^{B} T_{\mathrm{II}}$ be the coordinates of track points $T_{\mathrm{I}}$ and $T_{\mathrm{II}}$ in the slave manipulator coordinate system. The homogeneous transformation matrix ${ }^{\alpha} T_{\beta}$ of $4 \times 4$ represents point coordinates in rectangular coordinates as follows:

$$
{ }^{\alpha} T_{\beta}=\left[\begin{array}{cc}
R & E \\
0 & 1
\end{array}\right], \alpha=A 、 B, \beta=1 、 2 、 \text { I } \Pi \Pi
$$

where $R \in R^{3 \times 3}$ represents the rotation transformation matrix and $E \in R^{3 \times 3}$ represents the translation transformation matrix.

The relative pose constraint relation between track points $T_{1}$ and $T_{\mathrm{I}}$ is equal to that between track points $T_{2}$ and $T_{\mathrm{II}}$, so ${ }^{B} T_{\mathrm{II}}$ can be calculated according to ${ }^{A} T_{1}$, ${ }^{A} T_{2}$ and ${ }^{B} T_{\mathrm{I}}$. Let the homogeneous transformation matrix from the T1 manipulator base coordinate system to the main manipulator base coordinate system be 9 . Let the homogeneous transformation matrix from the $T_{\mathrm{I}}$ manipulator base coordinate system to the main manipulator base coordinate system be ${ }^{A} U_{B}$. The results are as follows:

$$
\begin{aligned}
& { }^{A} T_{\mathrm{I}}={ }^{A} U_{B} \cdot{ }^{B} T_{\mathrm{I}} \\
& { }^{A} T_{\mathrm{II}}={ }^{A} U_{B} \cdot{ }^{B} T_{\mathrm{II}}
\end{aligned}
$$

${ }^{A} T_{\mathrm{I}}$ and ${ }^{A} T_{\mathrm{II}}$ represent the coordinate representations of track points $T_{\mathrm{I}}$ and $T_{\mathrm{II}}$ in the base coordinate system of the main manipulator. In the base coordinate system of the main manipulator, $T_{1}$ and $T_{\mathrm{I}}$ are expressed as ${ }^{A} T_{1}$ and ${ }^{A} T_{\mathrm{I}}$. Let ${ }^{A} U_{1}$ be the homogeneous transformation matrix of $T_{1}$ to $T_{\mathrm{I}}$, then 


$$
{ }^{A} T_{1}={ }^{A} U_{1} \cdot{ }^{A} T_{\mathrm{I}}
$$

which can be obtained from Equation (2.4):

$$
{ }^{A} U_{1}={ }^{A} T_{1} \cdot\left({ }^{A} T_{\mathrm{I}}\right)^{-1}
$$

Substituting Equation (2.2) into Equation (2.5), we can obtain

$$
{ }^{A} U_{1}={ }^{A} T_{1} \cdot\left({ }^{B} T_{\mathrm{I}}\right)^{-1} \cdot{ }^{B} U_{A}
$$

where ${ }^{s b} H_{m b}$ represents the inverse matrix of ${ }^{m b} H_{s b}$ :

$$
{ }^{B} U_{A}=\left({ }^{A} U_{B}\right)^{-1}
$$

In the base coordinate system of the main manipulator, the coordinates of track points $T_{2}$ and $T_{\mathrm{II}}$ can be expressed as ${ }^{A} T_{2}$ and ${ }^{A} T_{\mathrm{II}}$. Set ${ }^{A} U_{2}$ as the homogeneous change matrix of $T_{2}$ to $T_{\text {II }}$, then

$$
{ }^{A} T_{2}={ }^{A} U_{2} \cdot{ }^{A} T_{\mathrm{II}}
$$

Substituting Equation (2.3) into Equation (2.8), we can obtain

$$
{ }^{A} T_{2}={ }^{A} U_{2} \cdot{ }^{A} T_{\mathrm{II}}={ }^{A} U_{2} \cdot{ }^{A} U_{B} \cdot{ }^{B} T_{\mathrm{II}}
$$

Since the pose constraint equation of $T_{1}$ and $T_{\mathrm{I}}$ is equal to that of $T_{2}$ and $T_{\mathrm{II}}$, we can obtain

$$
{ }^{A} U_{1}={ }^{A} U_{2}
$$

Substituting Equations (2.6) and (2.10) into Equation (2.9), we can obtain

$$
\begin{aligned}
{ }^{A} T_{2} & ={ }^{A} U_{2} \cdot{ }^{A} U_{B} \cdot{ }^{B} T_{\mathrm{II}} \\
& ={ }^{A} T_{1} \cdot\left({ }^{B} T_{\mathrm{I}}\right){ }^{-1} \cdot{ }^{B} U_{A} \cdot{ }^{A} U_{B} \cdot{ }^{B} T_{\mathrm{II}} \\
& ={ }^{A} T_{1} \cdot\left({ }^{B} T_{\mathrm{I}}\right){ }^{-1} \cdot{ }^{B} T_{\mathrm{II}} \\
{ }^{B} T_{\mathrm{II}} & ={ }^{B} T_{\mathrm{I}} \cdot\left({ }^{A} T_{1}\right){ }^{-1} \cdot{ }^{A} T_{2}
\end{aligned}
$$

Equation (2.12) shows that for any two points $T_{1}$ and $T_{2}$ on the main manipulator trajectory, under a specified coupling motion mode, the corresponding two points $T_{\mathrm{I}}$ and $T_{\mathrm{II}}$ on the slave manipulator trajectory and $T_{\mathrm{II}}$ on the slave manipulator trajectory can be calculated by ${ }^{A} T_{1}$ and ${ }^{A} T_{2}$ in the main manipulator base coordinate system and ${ }^{A} T_{\mathrm{I}}$ in the slave manipulator base coordinate system, respectively.

\subsubsection{Coupled motion coordination control}

Coupling motion control of multi-manipulators is investigated through a cooperative multi-manipulator handling task. In our double manipulator coordinated 
handling experiment, Robot 2 was set as the main manipulator and Robot 1 as the slave manipulator.

Assume that the main manipulator, Robot2, moves in a straight line from $T_{1}$ to $T_{2}$ and then to $T_{3}$ in its base coordinate system.

$$
T_{1}=\left[\begin{array}{cccc}
1 & 0 & 0 & 1700 \\
0 & -1 & 0 & 450 \\
0 & 0 & -1 & 30 \\
0 & 0 & 0 & 1
\end{array}\right] \quad T_{2}=\left[\begin{array}{cccc}
1 & 0 & 0 & 1500 \\
0 & -1 & 0 & 600 \\
0 & 0 & -1 & 130 \\
0 & 0 & 0 & 1
\end{array}\right] \quad T_{3}=\left[\begin{array}{cccc}
1 & 0 & 0 & 1100 \\
0 & -1 & 0 & 800 \\
0 & 0 & -1 & 90 \\
0 & 0 & 0 & 1
\end{array}\right]
$$

Then, the first point of the trajectory of Robot 1 can be determined as

$$
K=\left[\begin{array}{cccc}
1 & 0 & 0 & 1700 \\
0 & -1 & 0 & 50 \\
0 & 0 & -1 & 30 \\
0 & 0 & 0 & 1
\end{array}\right]
$$

The trajectory point matrix of the slave manipulator can be obtained by Equations (2-12), and there is no need to plan the slave manipulator's trajectory separately.

Figure 2.1 shows the four positions and postures in the handling process:

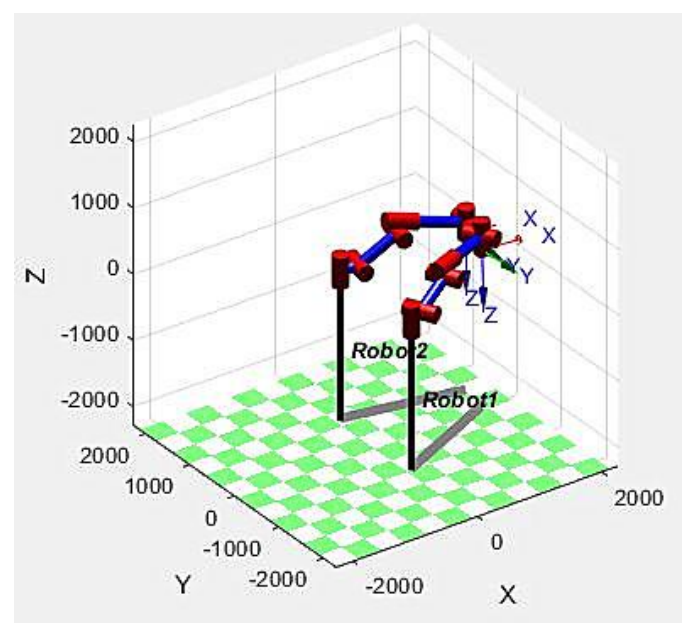

(a) Coupled handling pose 1

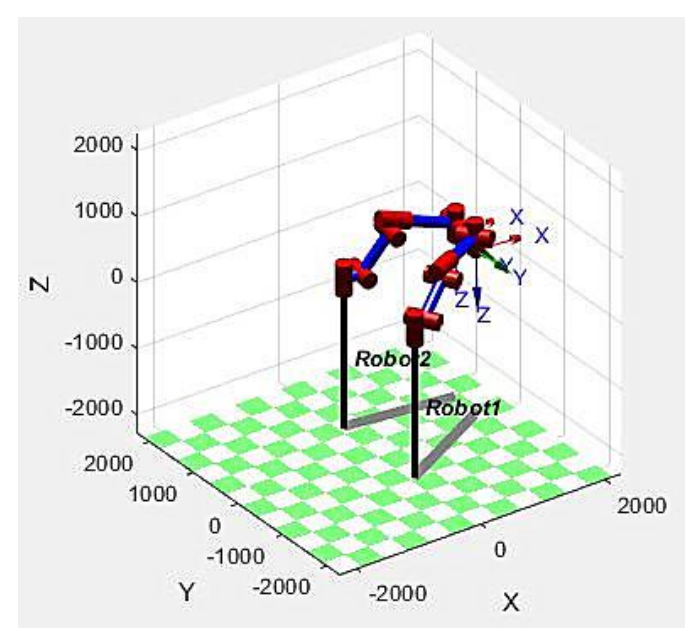

(b) Coupled handling pose 2 


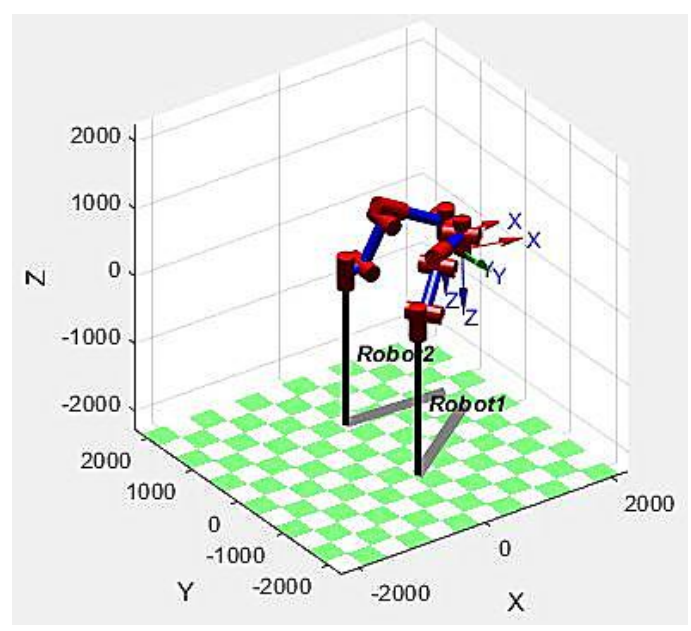

(b) Coupled handling pose 3

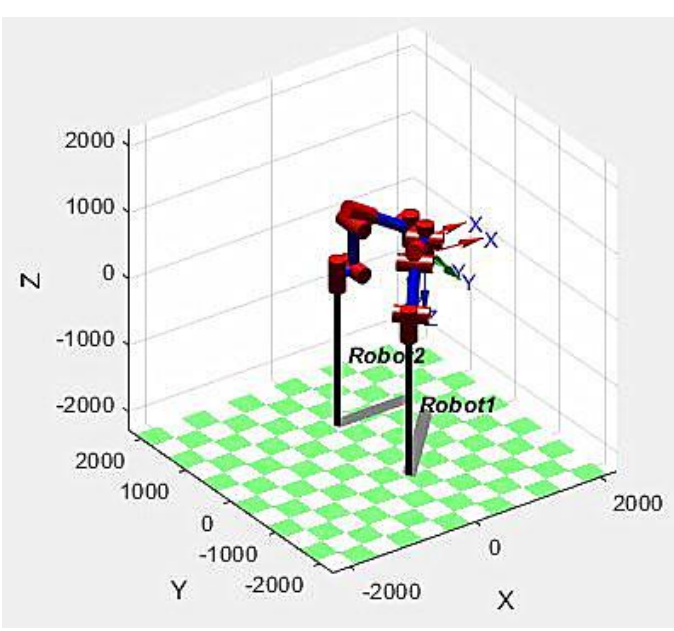

(d) Coupled handling pose 4

Figure 2.1 Coupling handling of cooperative multi-manipulator system

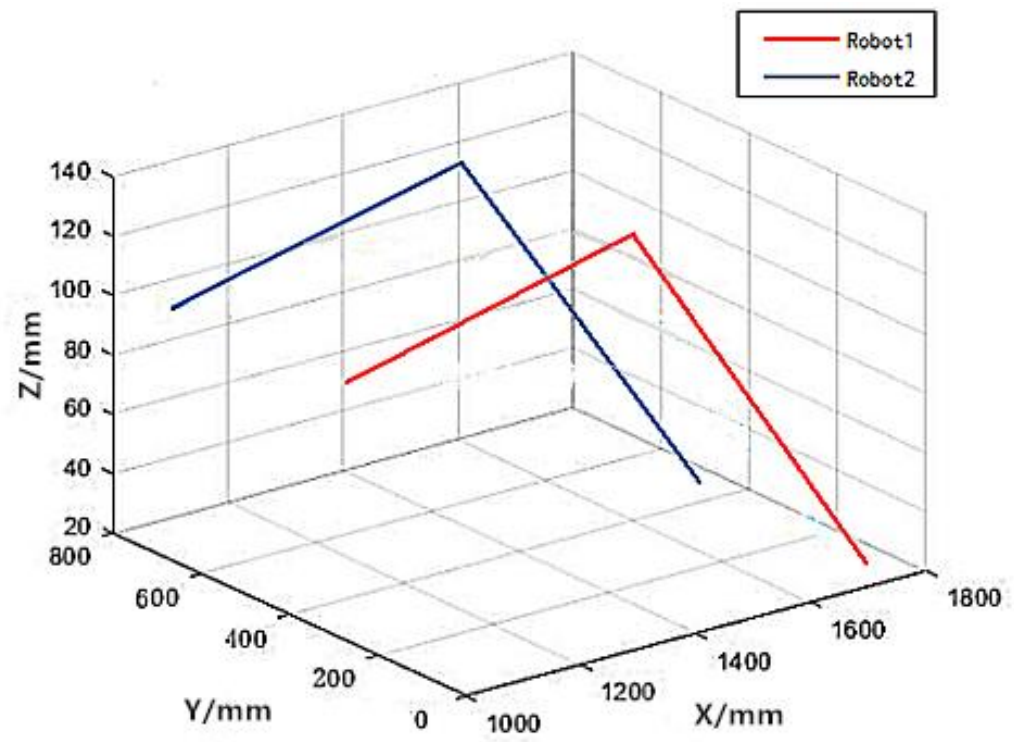

Figure 2.2 Coupling motion trajectory diagram

Figure 2.2 shows that the simulation trajectory of the coupled motion of the two manipulator is consistent with the planned trajectory, and the relative pose of the ends of the two manipulators always remains unchanged, indicating the correctness of the coupled motion algorithm.

\subsection{Multi-manipulator superposition motion coordination and constraint control}

\subsubsection{Superposition motion constraint algorithm}

In the main manipulator's base coordinate system, the coordinates of any point on the trajectory of the main manipulator are expressed as ${ }^{A} T_{1},{ }^{m} U_{A}(t)$ is the transformation matrix from the base coordinate system of the main manipulator to the reference coordinate system of the end-effector. ${ }^{A} T_{1}$ is the exact value of ${ }^{m} U_{A}(t)$ at 
time $t_{0}$, i.e.

$$
{ }^{A} T_{1}={ }^{m} U_{A}\left(t_{0}\right)
$$

$$
{ }^{m} U_{A}(t)={ }^{m} f(\theta(t)) \cdot{ }^{m f} U_{m}={ }^{A} T(t)
$$

where $\theta(t)$ represents the joint coordinate value vector of the manipulator at time $t,{ }^{m} f(\theta(t))$ represents the positive solution of the kinematics of the main manipulator, and ${ }^{m f} U_{m}$ represents the transformation matrix from the reference coordinate system of the end-effector of the main manipulator to the flange coordinate system of the main manipulator.

Suppose ${ }^{A} T_{\mathrm{I}}$ is the pose of any point on the trajectory of the slave manipulator in the base coordinate system of the master manipulator, then

$$
\begin{gathered}
{ }^{m} T_{\mathrm{I}}={ }^{m} U_{A}\left(t_{0}\right) \cdot{ }^{A} T_{\mathrm{I}} \\
{ }^{m} U_{A}(t)=\left({ }^{A} U_{m}(t)\right)^{-1}
\end{gathered}
$$

${ }^{m} U_{A}(t)$ is the time-varying matrix from the main manipulator's base coordinate system to the end-effector's reference coordinate system of the main manipulator. Substituting Equation (2.2) into Equation (2.15), we can obtain

$$
{ }^{m} T_{\mathrm{I}}={ }^{m} U_{A}\left(t_{0}\right) \cdot{ }^{A} U_{B} \cdot{ }^{B} T_{\mathrm{I}}
$$

${ }^{m} T_{\mathrm{I}}$ represents the trajectory of the slave manipulator in the reference coordinate system of the end-effector of the main manipulator. Equation (2.17) applies to any point on the trajectory of the slave manipulator within the entire cooperation cycle $\Delta t$ of the cooperative multi-manipulator system, namely,

$$
{ }^{m} T(t)={ }^{m} U_{A}(t) \cdot{ }^{A} U_{B} \cdot{ }^{B} T(t)
$$

It can be obtained from Equation (2.18): 


$$
{ }^{B} T(t)={ }^{B} U_{A} \cdot{ }^{A} U_{m}(t) \cdot{ }^{m} T(t)
$$

Equation (2.19) indicates that given the known motion trajectory of the master manipulator in its base coordinate system and the relative motion trajectory of the slave manipulator in the reference coordinate system of the end-effector of the master manipulator, the motion trajectory of the slave manipulator in its base coordinate system can be solved.

\subsubsection{Superimposed motion coordination control}

Superposition motion control is investigated by performing a cooperative multi-manipulator circle drawing task. Task requirements for coordinating circle drawing are as follows: a manipulator follows another manipulator and forms a certain relative motion trajectory, whose relative motion trajectory is three circles with a radius of $200 \mathrm{~mm}$. Set Robot2 as the master manipulator and its base coordinates as $(1500,0,0)$. It moves from $T_{1}$ to $T_{2}$ in its base coordinate system.

$$
T_{1}=\left[\begin{array}{cccc}
0 & 0 & -1 & -100 \\
0 & 1 & 0 & 0 \\
1 & 0 & 0 & 700 \\
0 & 0 & 0 & 1
\end{array}\right] T_{2}=\left[\begin{array}{cccc}
0 & 0 & -1 & 180 \\
0 & 1 & 0 & 0 \\
1 & 0 & 0 & 760 \\
0 & 0 & 0 & 1
\end{array}\right]
$$

Set Robot1 as the slave manipulator and its base coordinates as $(-1200,800,0)$. This coordination circle drawing task requires that the relative motion trajectory of the end-effector of Robot 1 on the reference plane of the end-effector of Robot 2 be a circle. Therefore, Robot 1 maintains the distance between the end-effector reference point and the end-effector of the main manipulator constant in its base coordinate system, and the $\mathrm{x}$-axis of the end-effector of the slave manipulator Robot1 is always perpendicular to the $x-y$ plane of the end-effector of the main manipulator Robot2. The actual motion of Robot1 consists of the following motion at a certain distance from the end-effector of Robot 2 and the relative motion in the reference plane of the end-effector of Robot2.

Because the collaborative task revealed that the relative distance between the end-effector of Robot1 and the end-effector of Robot2 is constant, the parametric equation of its relative motion trajectory is

$$
\left\{\begin{array}{l}
y=200 \cos \alpha \\
z=200 \sin \alpha
\end{array}\right.
$$


The trajectories of the two manipulators in Cartesian space were divided into 200 parts, and the relative trajectories of the manipulator Robot 1 during this period were three circles.

Figure 2.3 shows the base coordinate system of the two manipulators.

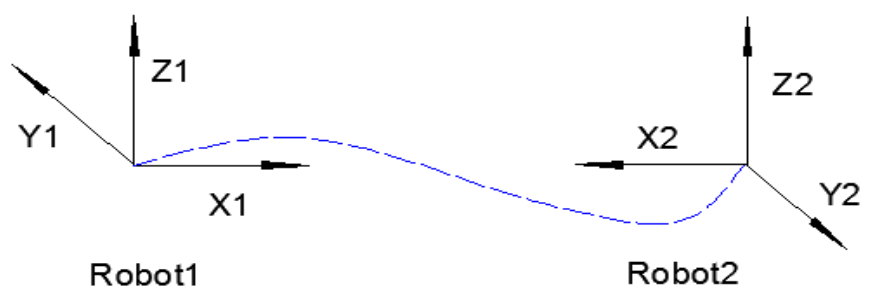

Fig. 2.3 Master/slave manipulator base coordinate system

Figure 2.3 shows that the base coordinate system of the slave manipulator rotates $180^{\circ}$ around the z-axis, the negative movement along the y-axis is $800 \mathrm{~mm}$ and the $\mathrm{x}$-axis moves a distance of $+2700 \mathrm{~mm}$ to complete the transformation to the base coordinate system of the main manipulator. The homogeneous transformation matrix $\mathrm{M}$ of the coordinate system relative to the base coordinate system of the main manipulator is as follows.

$$
M=\left[\begin{array}{cccc}
-1 & 0 & 0 & 2700 \\
0 & -1 & 0 & -800 \\
0 & 0 & 1 & 0 \\
0 & 0 & 0 & 1
\end{array}\right]
$$

According to Equation (2.19), the corresponding trajectory matrix of the manipulator in its base coordinate system can be obtained.

Then, inverse kinematics was used to solve the joint coordinates of the manipulator's. so that the joint coordinates of each point could be obtained. Trajectory simulation was conducted using MATLAB-Robot Toolbox. Figure 2.4 shows the four groups of poses in the task of drawing circles with dual manipulators. 


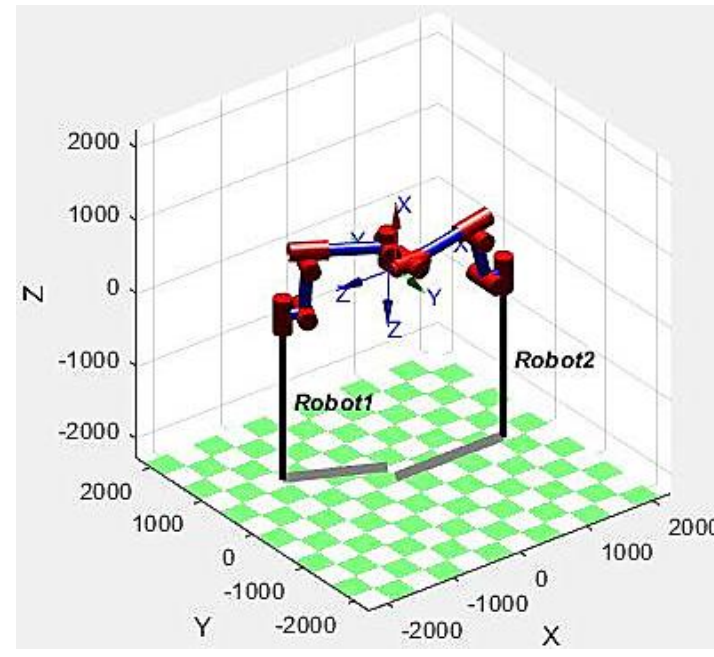

(a)Coordinated circle drawing - pose 1

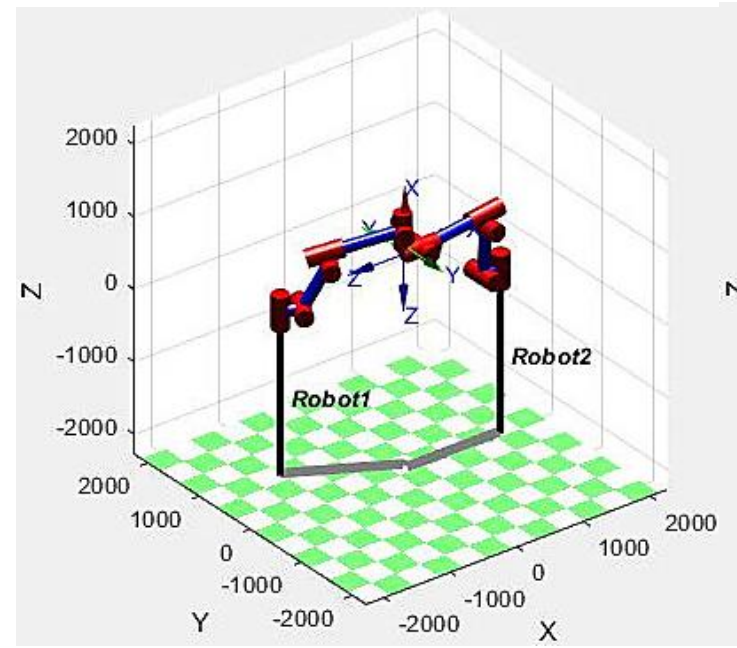

(c)Coordinated circle drawing - pose 3

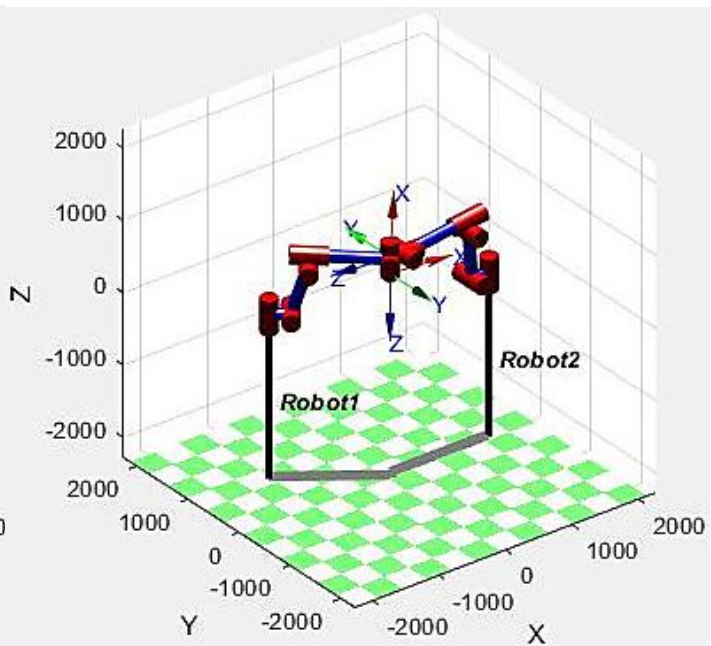

(b) Coordinated circle drawing - pose 2

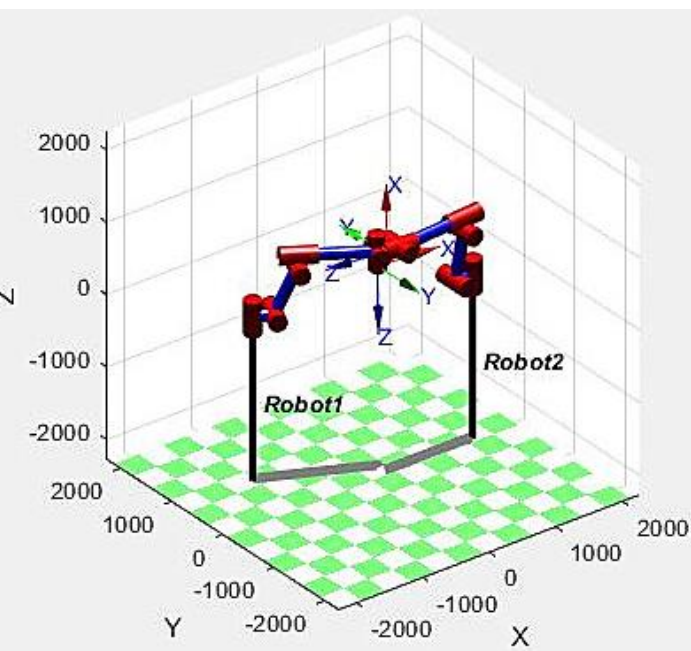

(d) Coordinated circle drawing - pose 4

Fig. 2.4 Simulation of cooperative multi-manipulator circle drawing task

In the circle drawing task of the master/slave manipulators, the slave manipulator moves with the master manipulator and draws a circle on a reference plane of the end-effector of the master manipulator. The relative trajectory is a circle, the actual trajectory of the master manipulator is a straight line and the actual trajectory of the slave manipulator is a spiral curve. Figure 2.5 shows an actual motion track of the master and slave manipulators. 


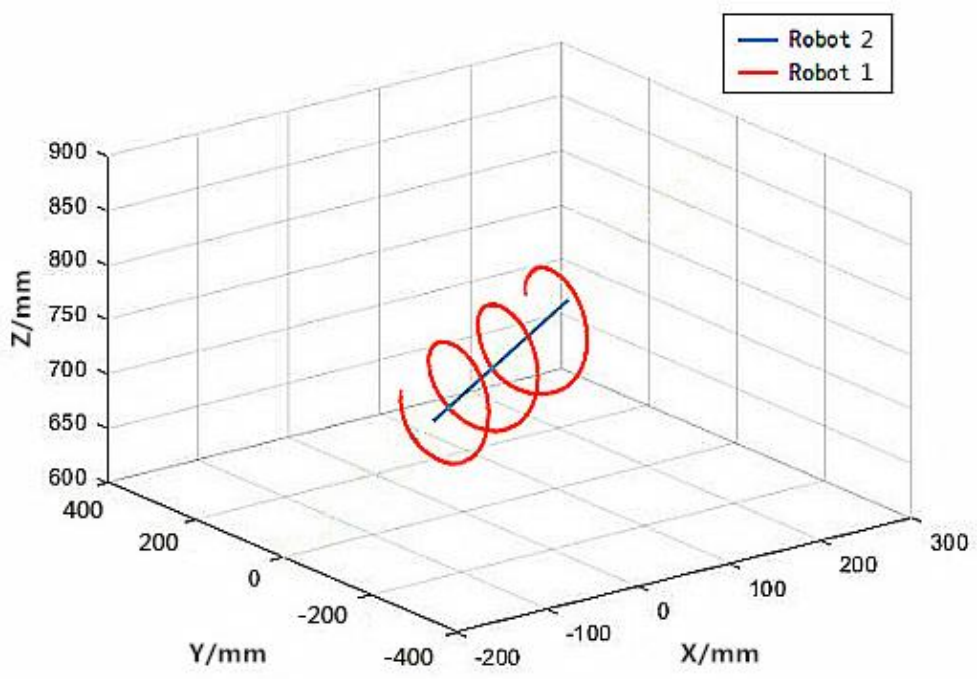

Figure 2.5 Actual motion trajectory of master/slave manipulators

According to Fig. 2.5, the actual trajectory of the manipulator obtained through trajectory simulation is a three-loop spiral, which conforms to the trajectory planning result and verifies the correctness of the multi-manipulator superposition motion algorithm.

3 Co-simulation of adaptive coordinated motion of a cooperative multi-manipulator system

Through MATLAB and ADAMS Co-simulation, the coordinated motion process of a multi-manipulator can be simulated more realistically. According to the multi-manipulator coupling and superposition motion coordination algorithm, trajectory planning is performed using MATLAB, and joint data are derived. A multi-manipulator simulation model is established in ADAMS, and then, the joint data are input into the model to create a spline curve, and the spline curve is used as the joint driving function to drive the virtual multi-manipulator model for coordinated movement.

\subsection{Coordinated handling simulation analysis}

The cooperative coupling motion of the multi-manipulator expands the manipulator's working range, allowing it to carry a large mass and volume of a workpiece, and its application prospect is broad. In this section, coupling motion simulation analysis is conducted through the cooperative handling of a cuboid load by multiple manipulators. Coordinated handling task requirements are as follows: the 
centre of mass of the load coordinated by multiple manipulators moves in accordance with the specified trajectory, and its attitude always remains unchanged in the process of load movement. According to the multi-manipulator trajectory coordination method, the trajectory coordination planning of the two manipulators is simulated in MATLAB, and the joint angle is calculated every $0.005 \mathrm{~s}$. In this simulation, the two manipulators act similarly when performing the coupled motion, so the angles of the joints corresponding to the two manipulators are also the same. Table 3.1 shows the joints of a manipulator at 2, 4, 6, 8 and $10 \mathrm{~s}$. For the rotation angle value, the unit of change angle of each joint in the table is degree.

Table 3.1 Coupling motion joint angle value

\begin{tabular}{cccccc}
\hline $\begin{array}{c}\text { Time } \\
\text { The joints }\end{array}$ & 2 & 4 & 6 & 8 & 10 \\
\hline $\mathrm{y}$ & 9.3727 & 27.009 & 27.602 & 21.381 & 19.264 \\
$\mathrm{~b}$ & 0.9312 & 8.4967 & 10.952 & 33.623 & 48.513 \\
$\mathrm{c}$ & -0.9390 & -9.1465 & -11.065 & -30.482 & -49.643 \\
$\mathrm{~d}$ & 0 & 0 & 0 & 0 & 0 \\
$\mathrm{e}$ & -0.0078 & -0.6498 & -0.1128 & 3.1404 & -1.1303 \\
$\mathrm{f}$ & 9.3727 & 27.009 & 27.602 & 21.381 & 19.264 \\
\hline
\end{tabular}

Spline curves were generated numerically for each joint, and spline curves were used as joint driving functions for the virtual multi-manipulator model. The simulation time is set as $10 \mathrm{~s}$. When the simulation begins, the two manipulators jointly carry the cuboid load, and the centroid of the cuboid moves from Point A to B and then to Point C. Figure 3.1 shows the simulation process is smooth without any delay. Four pose groups in the simulation process.
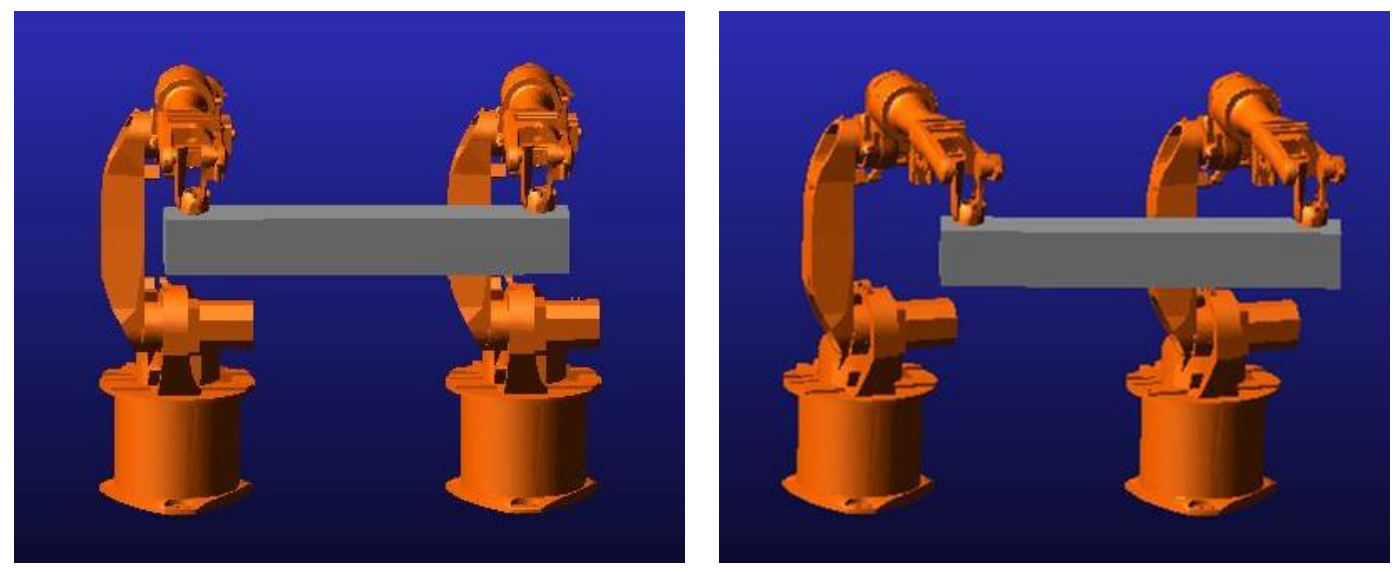

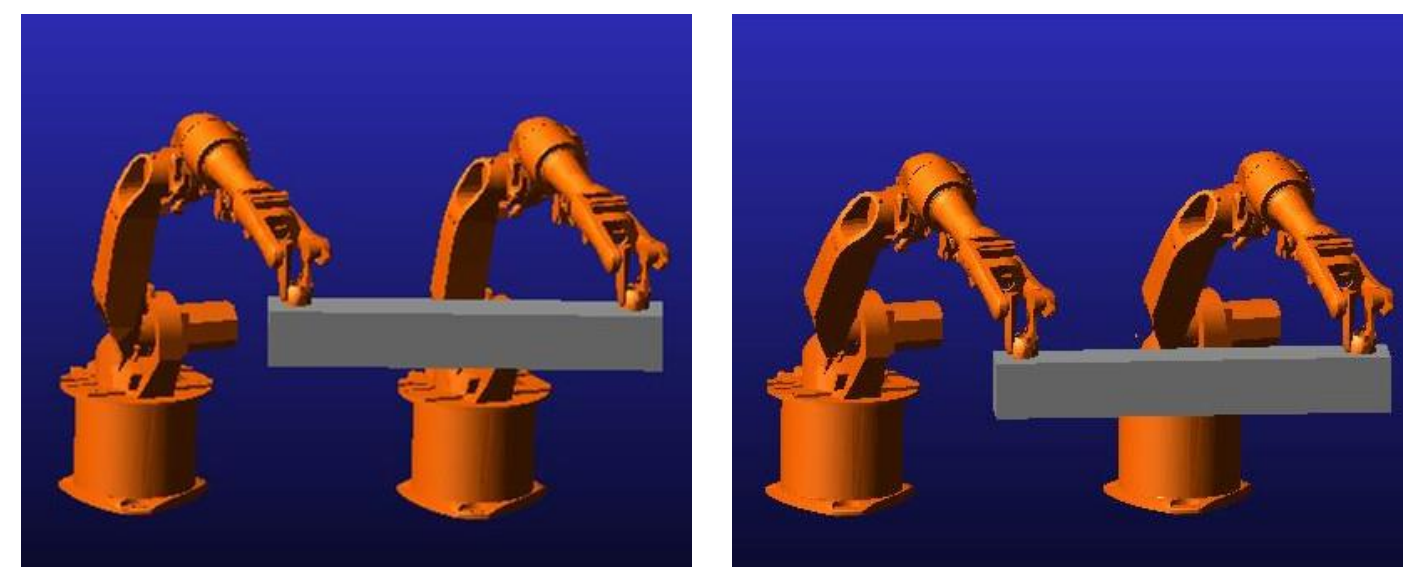

Fig. 3.1 Coupled motion simulation process

Figure 3.2 shows the angle changes of each joint of the two manipulators during the coupling motion simulation, where Fig. (a) illustrates the angle changes of each joint of the manipulator Robot 1 and Fig. (b) depicts the angle changes of each joint of the manipulator Robot2.

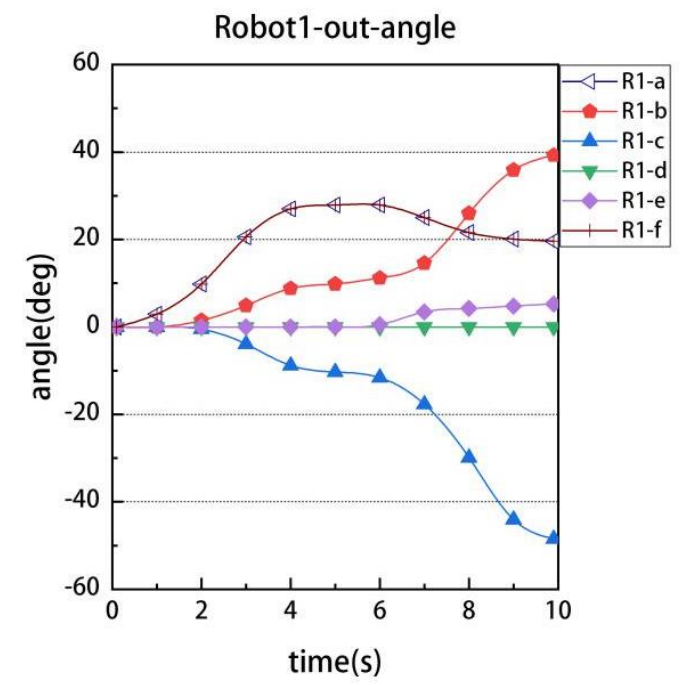

(a)

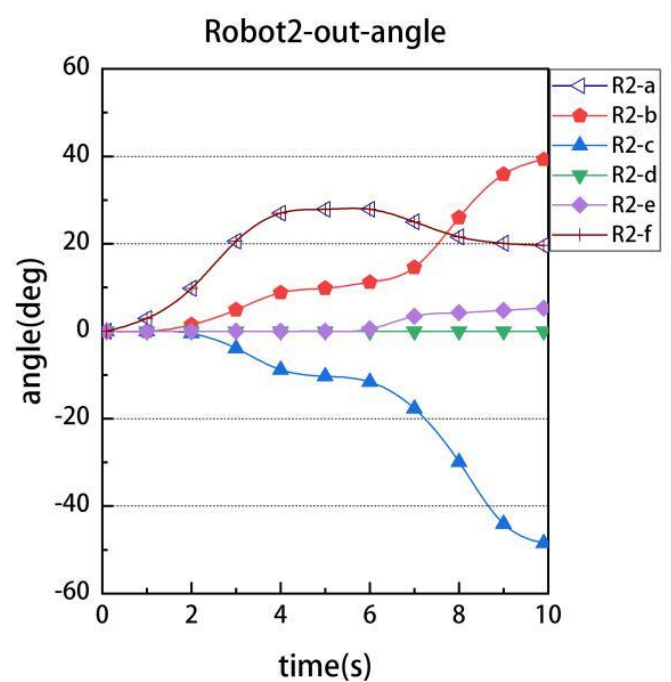

(b)

Figure 3.2 Angle change curve of coupled motion joint

Figure 3.3 shows the velocity changes of each joint during the coupling motion simulation of two manipulators. Figure (a) depicts the velocity changes of each joint of the manipulator Robot1, and Fig. (b) depicts the velocity changes of each joint of the manipulator Robot2. 


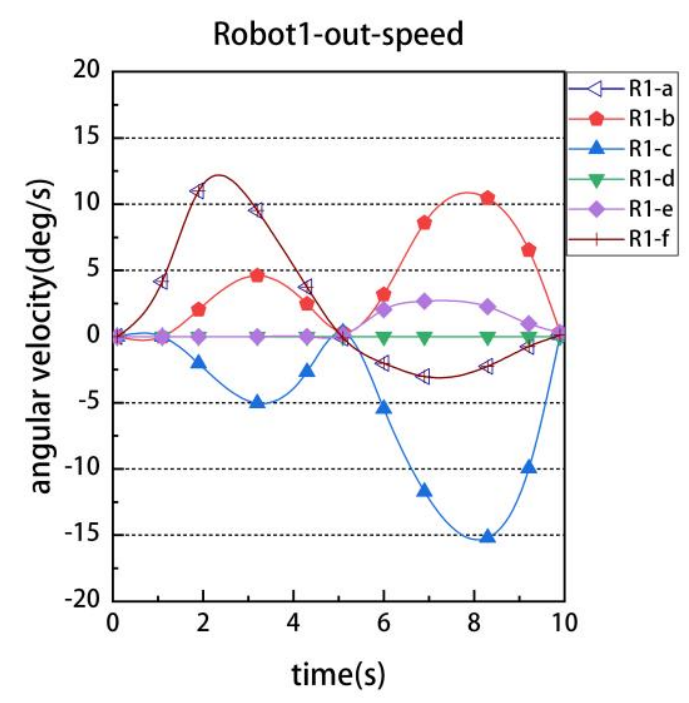

(a)

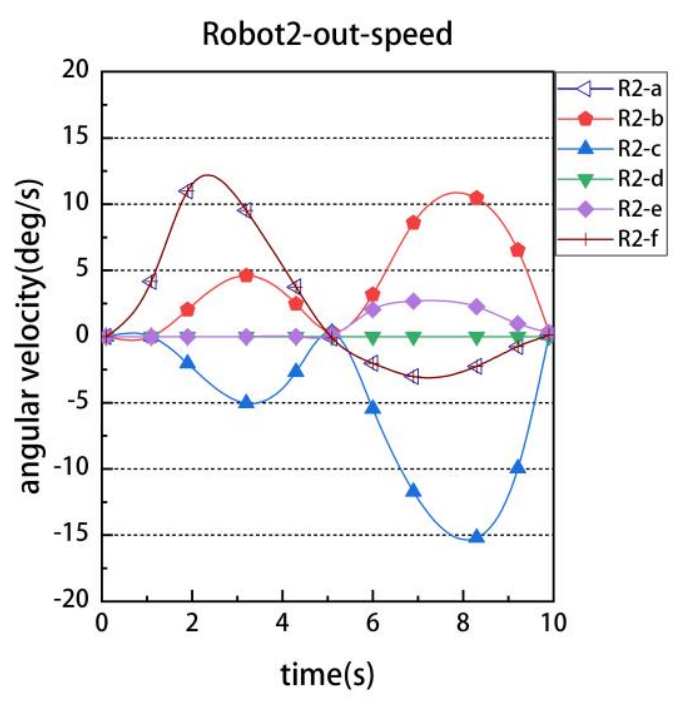

(b)

Figure 3.3 Speed change curve of coupled motion joint

Figures 3.2 and 3.3 depict that the angle and velocity curve changes of each joint are relatively smooth, indicating that during the simulation, the running speed of each joint of the two manipulators is relatively stable and the trajectory coordination effect is good.

\subsection{Simulation and analysis of coordinated circle drawing}

In this section, the simulation analysis of superposition motion is performed by drawing a circle through the collaboration of multiple manipulators. Coordinating the circle drawing task requires the end-effector of the manipulator Robot 2 to connect the drawing board. The drawing board moves in a straight line along the $\mathrm{x}$-axis, whereas the posture remains the same. It moves from Point D $(-100,1490,1250)$ to $E(100$, 1490, 1250). The end-effector of another manipulator, Robot1, is connected with the brush, which is always perpendicular to the drawing board. Draw a 330-mm-diameter circle on the drawing board by following the manipulator Robot 2 .

The spline curve was also used as the driving function in the superposition motion simulation. The trajectory coordination planning of the two manipulators was simulated in MATLAB, and the joint rotation angle was calculated every $0.005 \mathrm{~s}$. Table 3.2 shows the joint angle values of manipulator R1 at 2, 4, 6, 8 and $10 \mathrm{~s}$; Table 3.3 shows the joint angle values of manipulator R2 at 2, 4, 6, 8 and $10 \mathrm{~s}$. The unit of the change angle of each joint in the table is degree.

Table 3.2 Joint angle value of R1 superposition motion

\begin{tabular}{|c|c|c|c|c|c|}
\hline The joints & 2 & 4 & 6 & 8 & 10 \\
\hline $\mathrm{a}$ & -6.8409 & -29.381 & -37.607 & -31.065 & -28.256 \\
\hline
\end{tabular}




\begin{tabular}{cccccc} 
b & 1.7508 & 11.323 & 17.111 & 10.629 & 9.3386 \\
c & -7.4187 & -16.956 & -5.6284 & -4.5118 & -10.126 \\
d & -20.202 & -49.232 & -41.117 & -17.228 & 0 \\
e & -7.4209 & -25.708 & -19.549 & -4.1893 & -0.7876 \\
f & -4.5143 & -15.040 & -34.467 & -31.358 & -28.256 \\
\hline
\end{tabular}

Table 3.3 Joint angle value of R2 superposition motion

\begin{tabular}{cccccc}
\hline The joints & 2 & 4 & 6 & 8 & 10 \\
\hline a-2 & 1.5759 & 9.3727 & 20.415 & 27.008 & 28.245 \\
b-2 & 0.0275 & 0.9312 & 4.6155 & 8.4967 & 9.4032 \\
c-2 & -0.0275 & -0.9390 & -4.8074 & -9.1465 & -10.198 \\
d-2 & 0 & 0 & 0 & 0 & 0 \\
e-2 & 0 & -0.0078 & -0.1918 & -0.6498 & -0.7957 \\
f-2 & 1.5759 & 9.3727 & 20.415 & 27.008 & 28.245 \\
\hline
\end{tabular}

The spline curve is generated from the numerical value of each joint, and the spline curve is used as the joint driving function of the virtual multi-manipulator model. The simulation time was set to $10 \mathrm{~s}$. After the simulation started, the manipulator Robot 2 held the drawing board and moved the drawing board from Point $\mathrm{D}(-100,1490,1250)$ to $\mathrm{E}(100,1490,1250)$. The manipulator Robot1 held the paintbrush to draw a circle on the drawing board. The entire simulation process is smooth and free of lag. Figure 3.4 shows four pose groups in the simulation process.
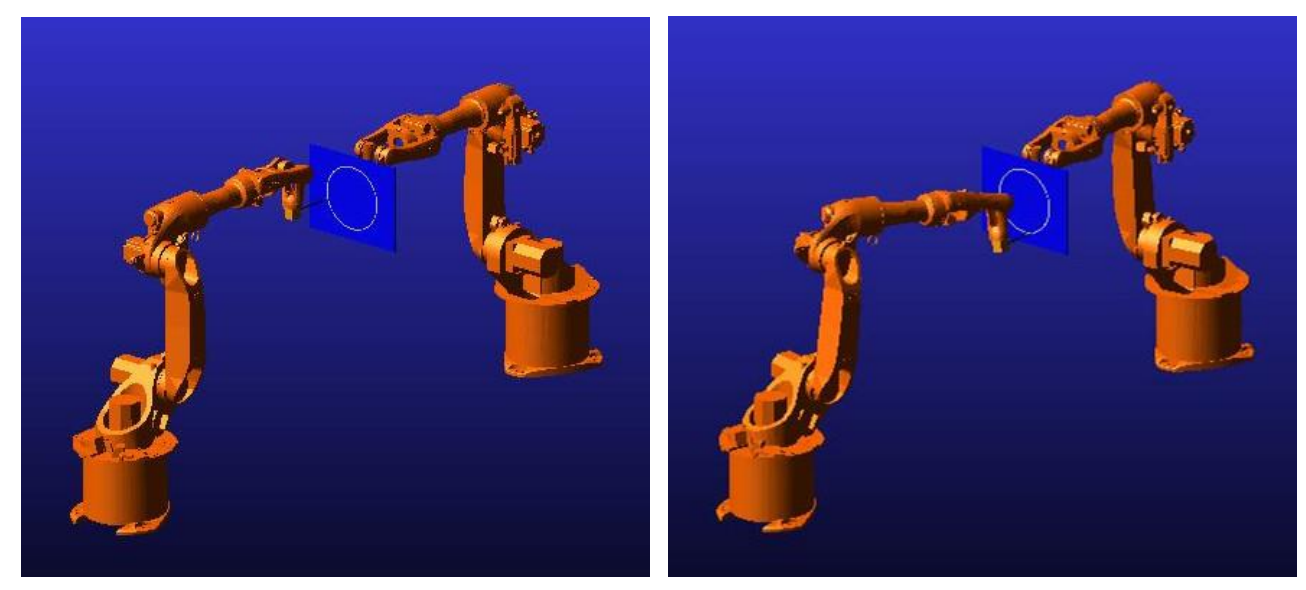

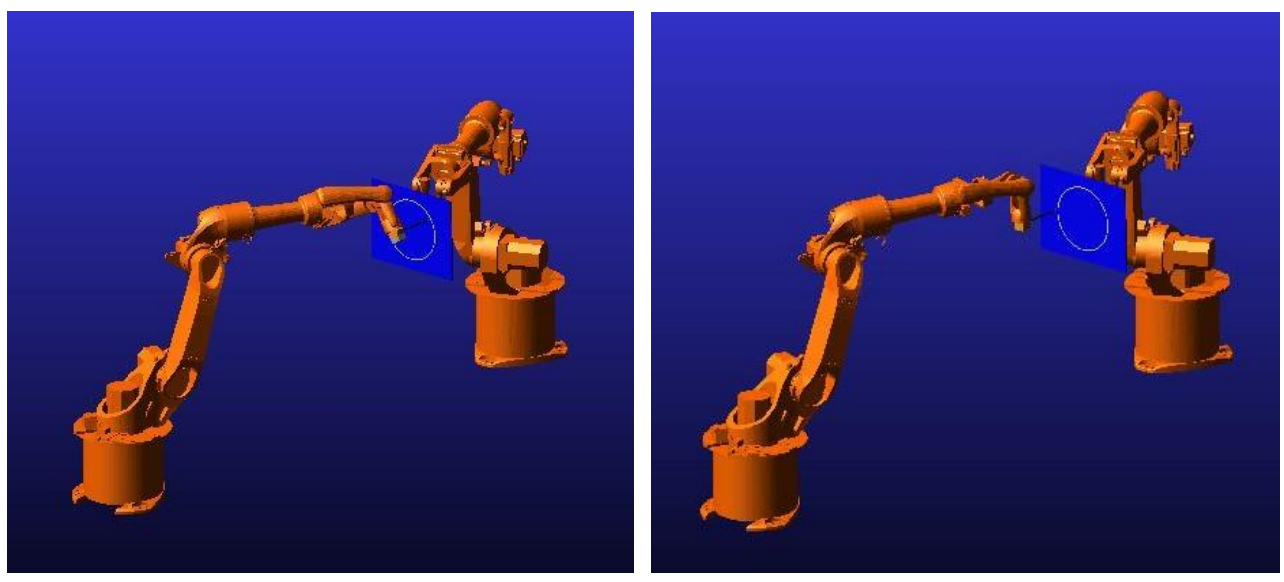

Figure 3.4 Superimposed motion simulation process

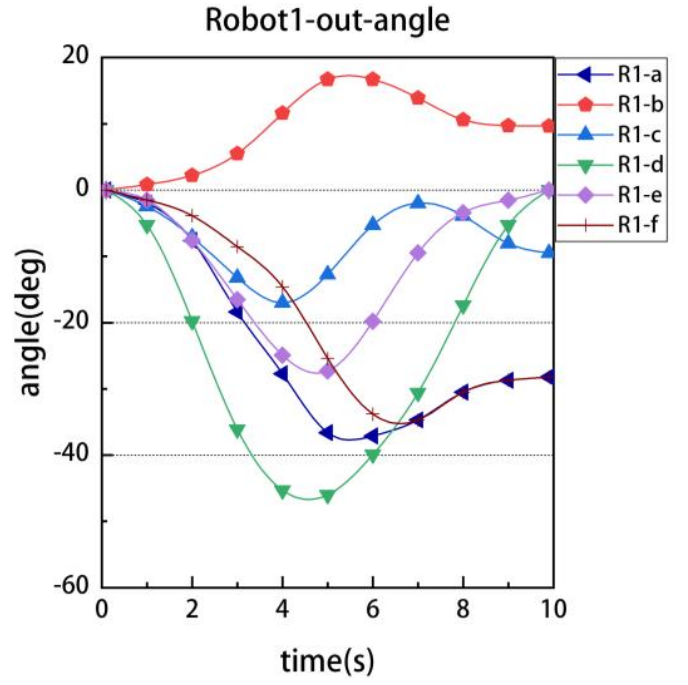

(a)

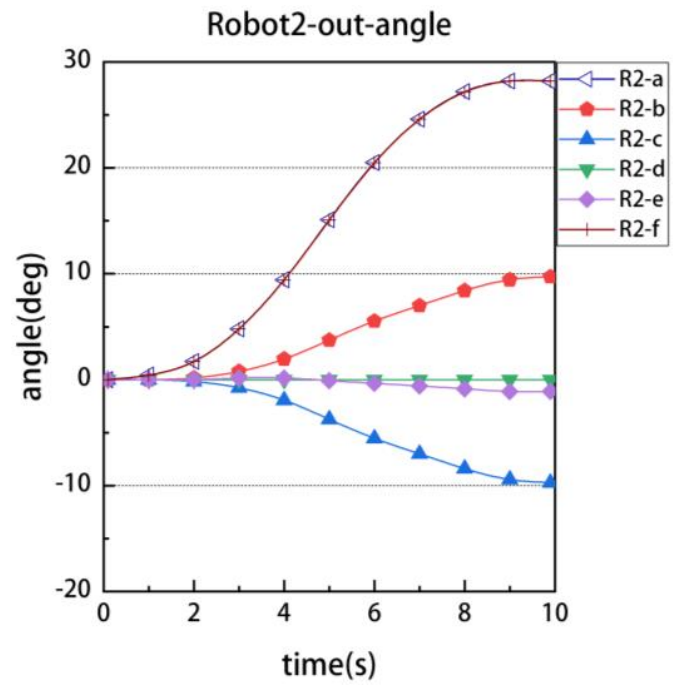

(b)

Fig. 3.5 Angle change curve of superimposed kinematic joint

Figure 3.5 shows the angle changes of each joint of the two manipulators during the superposition motion simulation, where Fig. (a) depicts the angle changes of each joint of the manipulator Robot1 and Fig. (b) depicts the angle changes of each joint of the manipulator Robot2.

Figure 3.6 shows the velocity changes of each joint in the superposition motion simulation process of the two manipulators. Figure (a) depicts the velocity changes of each joint of the manipulator Robot1, and Fig. (b) depicts the velocity changes of each joint of the manipulator Robot2. 


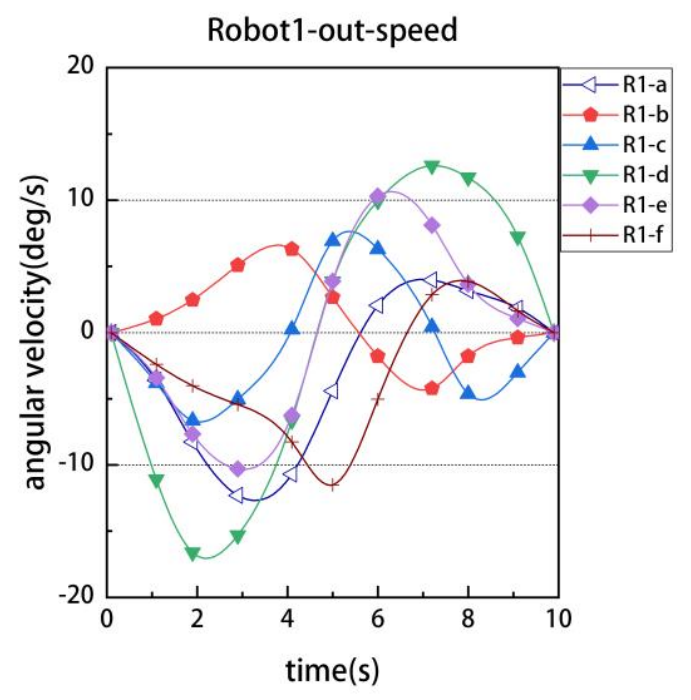

(a)

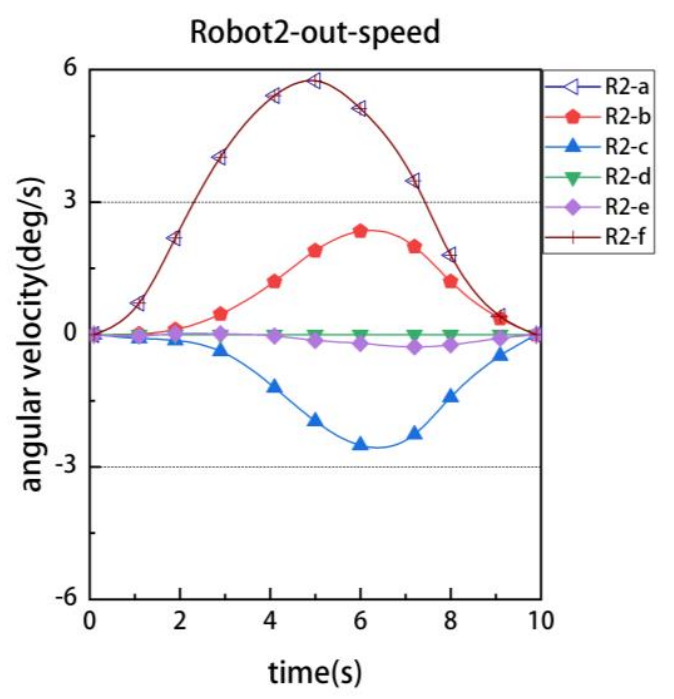

(b)

Fig. 3.6 Variation curve of the angular velocity of superimposed motion joint

Figures 3.5 and 3.6 show that the change of angle and velocity curves of each joint of the two manipulators is relatively smooth, indicating that during the superposition motion simulation process, the running speed of each joint of the two manipulators is relatively stable and the trajectory coordination effect is good.

\section{Experimental and analysis}

To verify the feasibility and accuracy of the coordinated motion algorithm of the cooperative multi-manipulator system, an experimental platform for the cooperative multi-manipulator system was developed to conduct experimental research on cooperative multi-manipulator circle drawing tasks. The cooperative multi-manipulator system's coordinated motion experiment platform is primarily composed of an upper computer (computer), a lower computer (speedgoat controller), servo drives and two mechanicals. As shown in Fig. 4.1, two 6-degree-of-freedom manipulators are used as experimental objects in the coordinated motion experiment of multi-manipulators. 


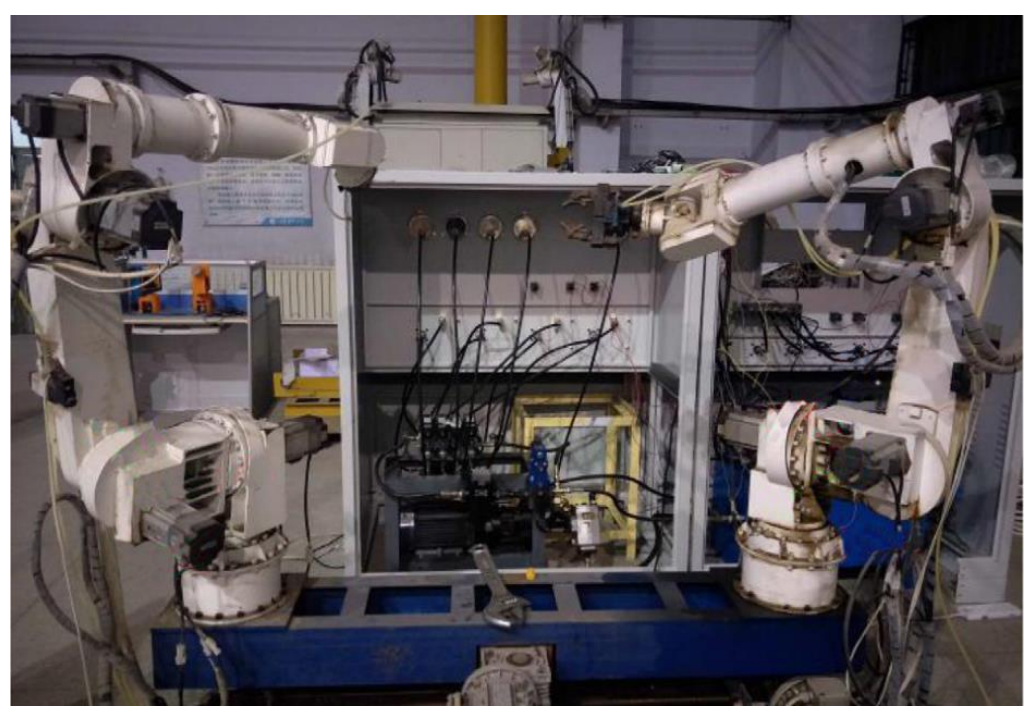

Fig. 4.1 Multi-manipulator experimental platform

In this study, the coordinated motion algorithm of the multi-manipulator is verified by the multi-manipulator coordinated circle drawing experiment. During the experiment, the end-effector of a manipulator, Robot2, was connected to the drawing board, which moved along a straight line with the same posture. The end-effector of another manipulator, Robot1, was connected with a brush following the movement of the manipulator, Robot2, and two circles with a diameter of $400 \mathrm{~mm}$ were drawn on the drawing board.

Before the experiment, the base coordinate system of the two manipulators should be calibrated first, and the homogeneous pose transformation matrix between the two manipulators base coordinate systems should be determined.

$$
{ }^{R 1} T_{R 2}=\left[\begin{array}{cccc}
-0.22494 & -0.97432 & 0.00959 & 886.610 \\
0.974365 & -0.22488 & -0.00698 & -1365.32 \\
0.004644 & -0.010914 & 0.99930 & 10.3318 \\
0 & 0 & 0 & 1
\end{array}\right]
$$

Then, trajectory planning is performed according to the coordinated motion method of the multi-manipulator. The servo driver is controlled by the controller to drive the joint of the manipulator, and the multi-manipulator is coordinated to draw a circle. After several experiments and debugging, the multi-manipulator coordinated circle drawing result is obtained, as shown in Fig. 4.2. 


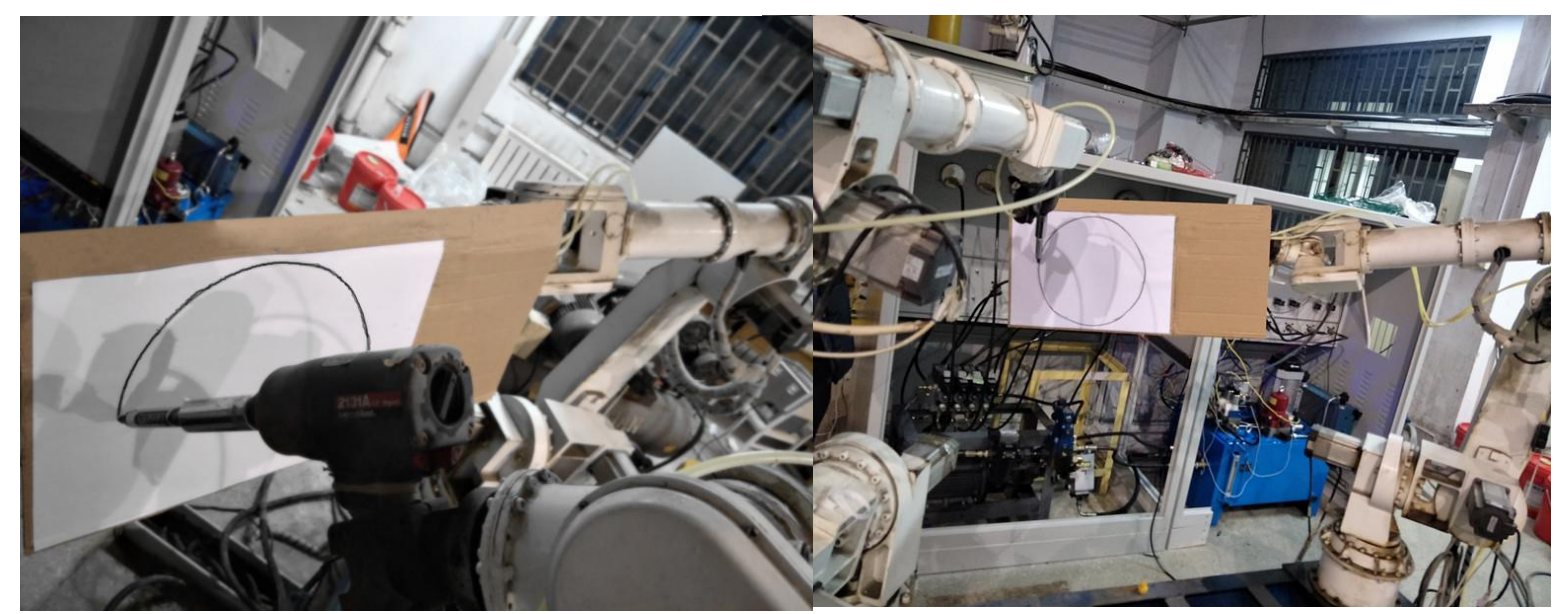

Fig. 4.2 Experiment of coordinated circle drawing with multiple manipulators

Figure 4.2 shows that after several experiments and debugging, the actual trajectory is consistent with the expected trajectory and can produce the basic shape required by the task, indicating the feasibility of the coordinated motion algorithm.

The experimental results will inevitably be affected by various factors, resulting in experimental errors. There were some trajectory tracking errors in the multi-manipulator coordinated circle drawing experiment, and the task curve was not sufficiently smooth. Therefore, this section analysed the experimental errors through the experimental results and investigated the accuracy of the coordination algorithm.

In the experiment of the multi-manipulator coordinated circular motion, the relative trajectory between the brush at the end-effector of manipulator R1 and the sketchpad clamped at the end-effector of manipulator R2 is circular. Because the actual motion trajectory at the end-effector of manipulator R2 is a straight line, whereas the actual motion trajectory at the end-effector of manipulator R1 is a spiral curve, the change of joint rotation angle is more complex, and the end-effector trajectory error is larger. Thus, the error of this experiment mainly comes from the error of manipulator R1. This section focuses on the analysis of the end-effector trajectory error of manipulator R1.

In the coordinated circular drawing movement, the eight groups of error values corresponding to the moment when the terminal position error of manipulator R1 varies greatly are shown in Table 4.1. The maximum position error in the $\mathrm{x}$-axis direction appears during the start-up phase, with a maximum error value of $-1.52 \mathrm{~mm}$. The maximum position error value in the y-axis direction appears at approximately $7.3 \mathrm{~s}$, with a maximum error value of approximately $1.58 \mathrm{~mm}$. The maximum position error value in the z-axis direction also appears at approximately $7.3 \mathrm{~s}$, with a maximum error value of approximately $2.13 \mathrm{~mm}$.

Table 4.1 End-effector position error of R1

\begin{tabular}{cccc}
\hline Time (s) & $\begin{array}{c}\text { X-position error } \\
(\mathrm{mm})\end{array}$ & $\begin{array}{c}\text { Y-position error } \\
(\mathrm{mm})\end{array}$ & $\begin{array}{c}\text { Z-position error } \\
(\mathrm{mm})\end{array}$ \\
\hline 0.01 & -1.521 & -0.615 & 2.102
\end{tabular}




\begin{tabular}{lccc}
0.22 & 0.423 & 1.033 & 1.038 \\
0.41 & -0.038 & 0.077 & 0.950 \\
2.25 & -0.192 & 0.696 & 1.006 \\
5.27 & 1.838 & 1.374 & 1.192 \\
7.32 & 1.385 & 1.579 & 2.131 \\
7.69 & 1.462 & 0.761 & 0.692 \\
9.55 & 1.521 & 0.739 & 1.661 \\
\hline
\end{tabular}

Figure 4.3 shows the position tracking error of the endpoint of manipulator R1. Comparing the actual position of the endpoint of the manipulator R1 with the expected position, the maximum errors in the $\mathrm{x}-, \mathrm{y}-$, and $\mathrm{z}$-axis directions in Cartesian space are $-1.523,1.579$ and $2.131 \mathrm{~mm}$, respectively. The end-effector position error of the manipulator R1 can meet the accuracy requirements in this coordinated circle drawing task.

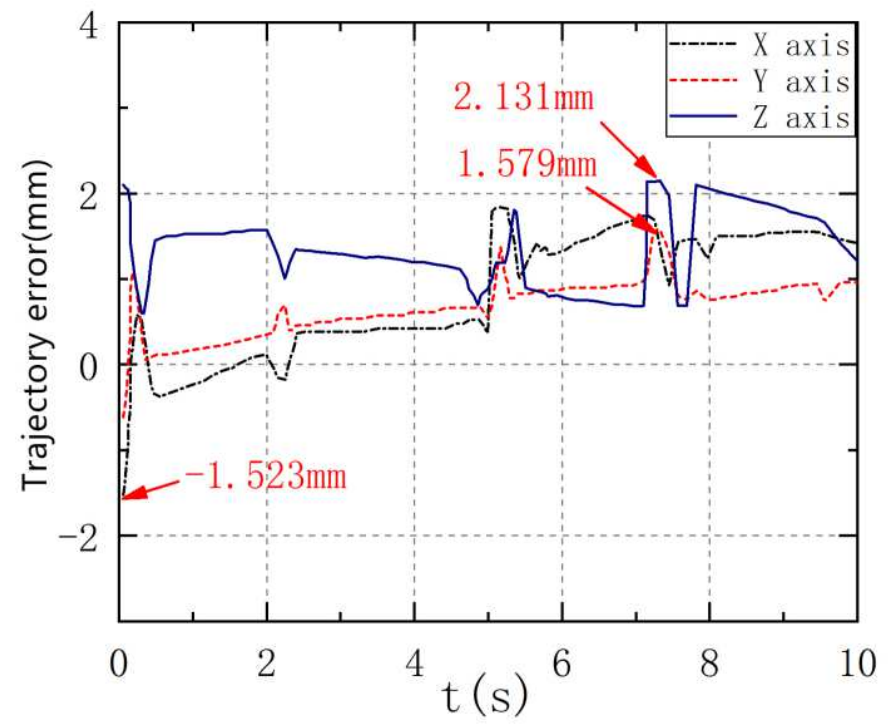

Figure 4.3 Position error of coordinated circle drawing motion

In this multi-manipulator coordinated motion experiment, there are also certain requirements for the posture of the end-effector of the manipulator. The brush at the end-effector of manipulator R1 and the drawing board at the end-effector of manipulator R2 always maintain a certain angle. When the posture error of the end-effector of manipulator R1 varies greatly, the corresponding eight groups of error values are shown in Table 4.2, and the unit of the angle of the change of each joint is degree. The maximum error of the rolling angle appears in the start-up stage, and the maximum error is approximately $0.176^{\circ}$. The maximum error of the pitch angle is approximately $5.3 \mathrm{~s}$, and the maximum error is approximately $0.067^{\circ}$. The maximum error of yaw angle is approximately $5.3 \mathrm{~s}$, and the maximum error is approximately $-0.053^{\circ}$.

Table 4.2 R1 end attitude error

\begin{tabular}{cccc}
\hline Time $(\mathrm{s})$ & $R z$-error $\left(^{\circ}\right)$ & $R y$-error $\left(^{\circ}\right)$ & $R x$-error $\left(^{\circ}\right)$ \\
\hline 0.01 & 0.176 & 0.044 & 0.046
\end{tabular}




$\begin{array}{lccc}0.22 & -0.078 & -0.011 & -0.035 \\ 0.41 & 0.101 & 0.033 & 0.023 \\ 2.25 & 0.004 & 0.046 & -0.014 \\ 5.36 & 0.053 & 0.067 & -0.053 \\ 7.32 & -0.012 & 0.016 & 0.037 \\ 7.69 & 0.074 & 0.030 & 0.015 \\ 9.55 & 0.056 & 0.067 & 0.045\end{array}$

Figure 4.4 shows the terminal attitude error of manipulator R1. Comparing the actual and expected attitude of the endpoint of manipulator R1, the maximum errors of rolling angle, pitch angle and yaw angle are $0.1761^{\circ}, 0.0674^{\circ}$ and $-0.0526^{\circ}$, respectively. In this coordinated circle drawing task, the terminal attitude error of manipulator R1 meets the accuracy requirements.

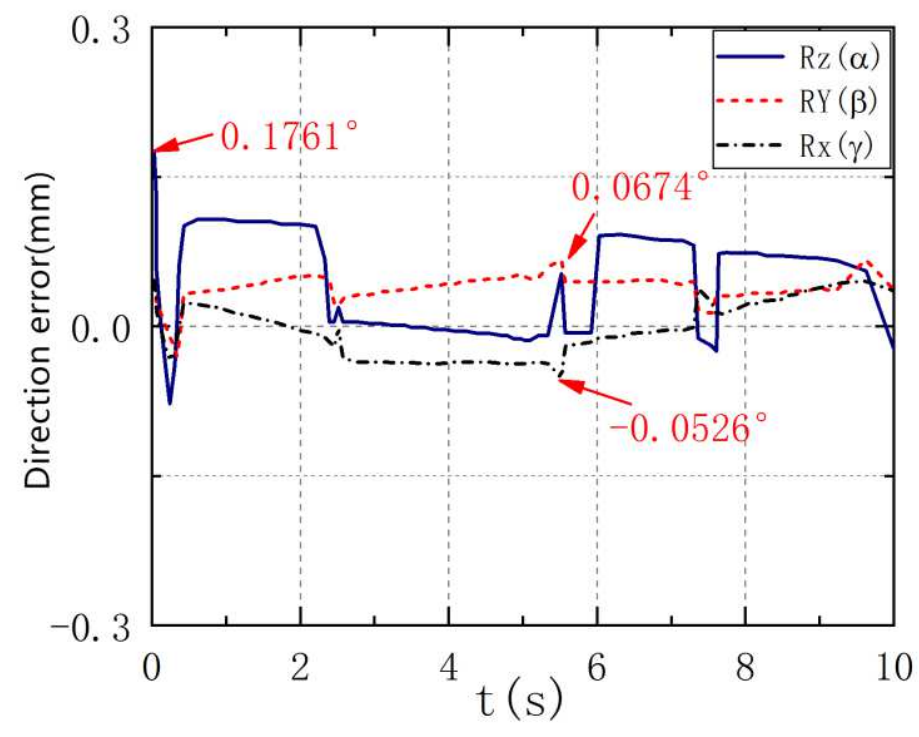

Fig.

4.4 Posture error of coordinated circular drawing motion

Figures 4.3 and 4.4 show that there is a large error value in the start phase of the manipulator. The main reason for the error at this stage is the start delay. Additionally, the error value varies significantly between 5 and $8 \mathrm{~s}$, and the cause of the error at this stage is more complicated. Analysing the error peak value, the maximum position error and the maximum attitude error can meet the requirements of coordinated motion accuracy.

Although error always exists in the entire coordinated motion and the error value varies frequently, the error value can be controlled in a certain range to meet the accuracy requirements, demonstrating the feasibility and accuracy of the adaptive coordinated motion algorithm of the multi-manipulator. 


\section{Conclusion}

(1) In this study, an adaptive coordinated motion constraint control scheme for a cooperative multi-manipulator system is proposed, and coordinated motions of the multi-manipulator system are divided into coupling and superposition motions while considering the kinematics constraints of the coordinated motion of the multi-manipulator.

(2) According to the adaptive trajectory coordination constraint control algorithm of multi-manipulator, there is no need to plan the motion of each manipulator in the cooperative multi-manipulator system; only the trajectory of the master manipulator has to be determined, and the trajectory of the other (slave) manipulator can be obtained automatically by the adaptive trajectory constraint algorithm. Using this method to coordinate the movement of the cooperative multi-manipulator system achieves a better coordination effect. When there is an external disturbance, only the main manipulator needs to be adjusted in motion, and other manipulators can be automatically adjusted using the adaptive motion constraint algorithm.

(3) This study uses multi-manipulator coordinated handling and circle drawing to investigate coupled and superimposed motions, respectively, which verifies the correctness of the control scheme proposed in this study.

(4) In this study, MATLAB and ADAMS are used for the joint simulation analysis of the multi-manipulator. The simulation results show that the proposed adaptive trajectory coordinated motion constraint control scheme has a good coordination effect, and the cooperative multi-manipulator system can always maintain a coordinated motion relationship during movement.

(5) Finally, a multi-mechanical coordinated motion experimental platform is developed to conduct the multi-manipulator coordinated circle drawing experiment. The experimental results show that the proposed control scheme has a good coordination control effect and strong self-adaptive ability. 


\section{Declarations}

a. Funding (information that explains whether and by whom the research was supported)

This work was supported by the National Natural Science Foundation of China (Grant No. 51305241), the Natural Science Foundation of Shandong province (Grant No. ZR2018MEE022), and the Youth Entrepreneurship Fund of Shandong Higher Education Institutions (Grant No. 2019KJB015).

b. Conflicts of interest/Competing interests (include appropriate disclosures)

The authors have no financial or proprietary interests in any material discussed in this article.

c. Availability of data and material (data transparency)

The data of this study cannot be shared publicly.

d. Code availability (software application or custom code)

Not applicable.

e. Ethics approval (include appropriate approvals or waivers)

Not applicable.

f. Consent to participate (include appropriate statements)

Not applicable.

g. Consent for publication (include appropriate statements)

This article agrees to be published.

h. Authors' contributions (optional: please review the submission guidelines from the journal whether statements are mandatory)

In this study, the coordinated motion of a multi-manipulator is divided into two types: coupling and superposition motions. An adaptive coordinated motion constraint scheme is proposed for the two different motion forms. Solve the problem of coordinated motion control of multi-manipulator cooperation system.

\section{References}

[1] Ning Z . Typical mechanical arm research status were reviewed[J]. Information Recording 
Materials, 2018.

[2] Lindner T , Milecki A, Wyrwa D .Positioning of the Robotic Arm Using Different Reinforcement Learning Algorithms[J]. International Journal of Control Automation and Systems, 2021(2).

[3] Li D , Qi F , Dai X . Research status of multi-robot systems task allocation and uncertainty treatment[J]. Journal of Physics Conference Series, 2017, 887(1):012081.

[4] Ni J , Shi H , Wang M . Disturbance Observer-Based Cooperative Learning Tracking Control for Multi-Manipulators[C]// 20207th International Conference on Information, Cybernetics, and Computational Social Systems (ICCSS). 2020.

[5] Lindner T, Milecki A , Wyrwa D . Positioning of the Robotic Arm Using Different Reinforcement Learning Algorithms[J]. International Journal of Control Automation and Systems, 2021(2).

[6] Liu, Xiangyu, Zhang, et al. Hybrid adaptive impedance-leader-follower control for multi-arm coordination manipulators[J]. Industrial Robot: An International Journal, 2016.

[7] Slota A . Bezier Curve Based Programmed Trajectory for Coordinated Motion of Two Robots in Cartesian Space[J]. Applied Mechanics and Materials, 2014, 555:192-198.

[8] Muscio G , Pierri F, Trujillo M A, et al. Experiments on coordinated motion of aerial robotic manipulators[C]// 2016 IEEE International Conference on Robotics and Automation (ICRA). IEEE, 2016.

[9] Zhai F , Wei L, Han S, et al. Vertical lifting control of forging manipulator clamp based on multi-actuator coordination[J]. Forging \& Stamping Technology, 2017.

[10] Zhang Haitao, Tang Licai, et al. Path planning algorithm for dual arm coordinated operation of space manipulator [J]. control engineering ,2015, 22(6):1028-1033.

[11]Hou Y, Wang T, Yue J, et al. Path Planning for Dual-robot Coordinate Welding Based on Multi-objective Genetic Algorithm[J]. Zhongguo Jixie Gongcheng/China Mechanical Engineering, 2018, 29(16):1984-1989.

[12] Zhang $\mathrm{T}$, Fan $\mathrm{O}$. Offline motion planning and simulation of two-robot welding coordination[J]. Frontiers of Mechanical Engineering, 2012, 7(001):81-92.

[13] Park H A , Lee C.Dual-arm coordinated-motion task specification and performance evaluation[C]. IEEE/RSJ International Conference on Intelligent Robots \& Systems. IEEE, 2016:929-936.

[14] Davidson J R , Hohimer C J , Mo C , et al. Dual Robot Coordination for Apple Harvesting[C]// Asabe International Meeting. 2017..

[15] Wang X , Chen L.A Vision-Based Coordinated Motion Scheme for Dual-Arm Robots[J]. Journal of Intelligent \& Robotic Systems, 2020, 97(12).

[16] Wang Z , Gan Y , Dai X . Human-Robot Distance Modeling and Minimum Distance Iterative Algorithm in Collaboration Environment[J]. Jiqiren/Robot, 2018, 40(4):413-422.

[17] Yu Guang Dong, Chen Qi. Kinematics analysis of dual robot coordination[J]. Manufacturing automation, 2016, 038(002):63-66.

[18] He Bin Bin, Huang Qing Dan, et al. Research on dual robot coordinated joint control based on task priority selection method [J]. Machine tool and hydraulic, 2019 (15).

[19] Tian Y, Wang B , Liu J , et al. Stiffness modeling and analysis of a multiple coordinated robot system[J]. International Journal of Advanced Manufacturing Technology, 2018, 94(1):1-12. 